\title{
Aqueous two-phase system-mediated antibody micropatterning enables multiplexed immunostaining of cell monolayers and tissues
}

\author{
John P. Frampton ${ }^{1}$, Michael Tsuei ${ }^{1}$, Joshua B. White ${ }^{1}$, Abin T. Abraham ${ }^{1}$ and Shuichi Takayama ${ }^{1,2}$ \\ ${ }^{1}$ Department of Biomedical Engineering, University of Michigan, Ann Arbor, MI, USA \\ ${ }^{2}$ Department of Macromolecular Science and Engineering, University of Michigan, Ann Arbor, MI, USA
}

Conventional immunostaining methods consume large quantities of expensive antibodies and are limited in terms of the number of antigens that can be detected from a single sample. In order to achieve multiplexed immunostaining, we micropatterned antibodies using aqueous two-phase systems (ATPS) formed from polyethylene glycol (PEG) and dextran. Multiple antigens can be detected on a single fixed sample by incorporating antibodies within dextran solutions, which are then patterned by micropipetting at specific sites on the sample in a solution of PEG. The antibodies are retained within the dextran phase due to biomolecular partitioning, allowing multiple protein markers to be visualized simultaneously by way of chromogenic, chemiluminescent, or immunofluorescent detection. This aqueous two-phase system-mediated antibody micropatterning approach allows antibody dilutions to be easily optimized, reduces the consumption of expensive primary antibodies and can prevent antibody cross-reactions, since the antibodies are retained at separate sites within the dextran microdroplets.

$\begin{array}{ll}\text { Received } & 24 \text { APR } 2014 \\ \text { Revised } & \text { 05 JUN } 2014 \\ \text { Accepted } & 18 \text { JUL } 2014 \\ \text { Accepted } & \\ \text { article online } & 21 \text { JUL } 2014\end{array}$

Supporting information available online

Keywords: Antibody micropatterning · Aqueous two-phase system · Immunohistochemistry · Multiplex immunostaining

\section{Introduction}

Immunostaining, one of the most frequently used techniques in the biomedical sciences, is typically performed by incubating fixed cells or tissue sections in solutions containing primary antibodies that recognize specific antigens. Labeled secondary antibodies that recognize the primary antibodies are then used to indirectly visualize the antigens. For decades, this strategy has provided valuable information about protein expression and local-

Correspondence: Prof. Shuichi Takayama, Biointerfaces Institute, 2800 Plymouth Road, NCRC Building 10, Ann Arbor, MI 48103, USA E-mail: takayama@umich.edu

Abbreviations: ATPS, aqueous two-phase system; CK7, cytokeratin 7; DMEM, Dulbecco's modified Eagle's medium; DRG, dorsal root ganglion; Ecad, E-cadherin; ELISA, enzyme-linked immunosorbent assay; FBS, fetal bovine serum; FITC-WGA, fluorescein isothiocyanate-wheat germ agglutinin; H2B, histone 2B; HBSS, Hank's balanced salt solution; HRP, horse radish peroxidase; $\mathrm{PEC}$, polyethylene glycol; SMA, smooth muscle actin; TRITC, tetramethylrhodamine isothiocyanate ization to biologists and clinicians [1, 2]. However, conventional immunostaining methods consume large quantities of expensive antibodies. In addition, the number of antigens that can be detected on one sample is often limited by the number of available detection channels (typically four or less for immunofluorescence and only one for chromogenic and chemiluminescent detection). Furthermore, when multiple primary antibodies are used together in solution the results can be confounded by higher background signals and antibody cross-reactions.

Because of these limitations, there has been increasing demand for more efficient multiplexed immunostaining methods. Quantum dots [3] and other advanced imaging and probing methods [4] can increase the number of antigens detected by fluorescence, but require either specific combinations of antibodies optimized to prevent cross-reactivity or iterative inactivation of the fluorescent probes. Antigen transfer methods, such as the layered peptide array [5], offer another promising approach to multiplexed immunostaining. However, these methods require sequential transfer of antigens to multiple sub- 
A

Step 1: Fix and Prepare Sample for Immunolabeling

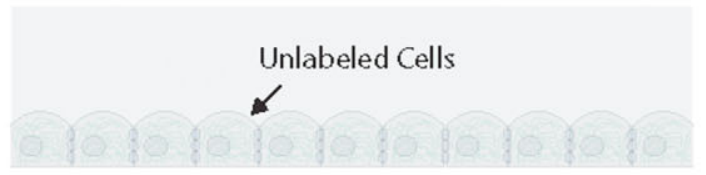

Step 2: Pattern Antibodies in ATPS

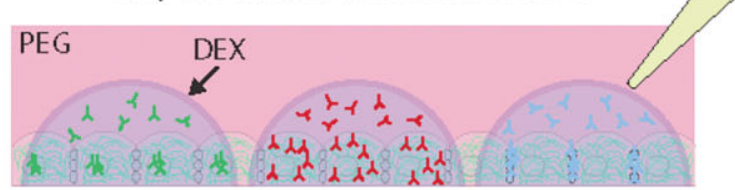

Step 3: Wash, Label with Secondary Antibodies and Prepare for Imaging

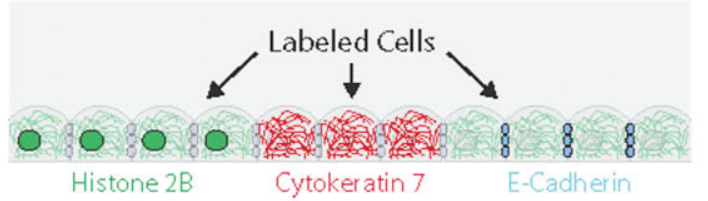

B
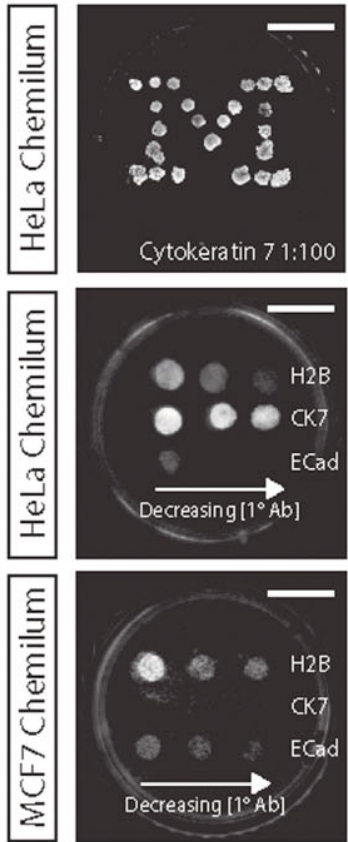

C
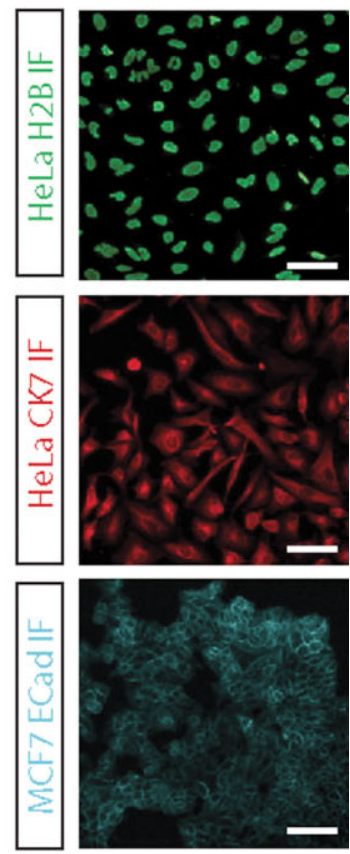

Figure 1. Multiplexed immunostaining of cell monolayers. (A) Aqueous two-phase system-(ATPS)mediated multiplexed immunostaining uses ATPSs composed of PEG and dextran to micropattern primary antibody solutions on the surface of the sample. Apart from the primary antibody incubation step, we follow standard immunostaining procedures. (B) Chemiluminescence detection of cytokeratin 7 (CK7), histone 2B (H2B), and E-cadherin (ECad) in antibody-micropatterned HeLa and MCF7 cell monolayers. The top image shows a HeLa monolayer immunostained in a "Michigan M" pattern using 23 dextran droplets containing 1:100 anti-CK7 antibody. The middle and bottom images show cell type-specific staining for H2B (control), CK7 and Ecad in HeLa cells and MCF7 cells, respectively. From left to right, the antibody dilution were 1:100, 1:400, and 1:800 for the anti-H2B antibody, 1:100, 1:600, and 1:1000 for the anti-CK7 antibody and 1:100, 1:600, and 1:1500 for the anti-ECad antibody. The spacing between the primary antibody spots can be estimated from the scale bars, which are $\sim 10 \mathrm{~mm}$. (C) Immunofluorescence detection of antigens at dextran/antibody micropatterned spots for H2B (top, 1:1000 dilution), CK7 (middle, 1:100 dilution), and ECad (bottom, 1:400 dilution). Scale bars are $\sim 50 \mu \mathrm{m}$.

strates and may not be suitable for imaging subcellular localization of proteins. Microfluidic methods [6, 7] can be used to deliver small volumes of reagents to precise regions of a sample; however, they require specialized expertise and equipment, making them cumbersome to implement in laboratories and clinics.

We present an approach that takes advantage of the phase separation of polyethylene glycol (PEG) and dextran [8] solutions to enable micropatterning of antibodies directly on cell cultures and tissue samples using easily accessed tools, such as micropipettors. We previously demonstrated that dextran-micropatterning can confine a variety of reagents, including DNA [9], enzymes [10], and antibodies [11,12] for biotechnological applications ranging from gene delivery to multiplexed ELISA. The ATPS-mediated antibody micropatterning procedure for multiplexed immunostaining follows a workflow similar to other standard immunostaining procedures, with the exception that the primary antibodies are applied in dextran microdroplets to samples immersed in PEG (Fig. 1A). This simple strategy for applying the primary antibodies allows multiple antigens to be detected on a single sample, while consuming very small antibody quantities (less than $2 \mu \mathrm{L}$ of diluted antibody per spot). It also prevents antibody cross-reactions, because biomolecular partitioning of the antibodies to dextran keeps the antibodies spatially separated.

\section{Materials and methods}

\subsection{HeLa and MCF7 cells culture}

HeLa cells and MCF7 cells (ATCC: HTB-22; Lot: 5105358) were obtained from collaborators at University of Michigan and cultured in a humidified incubator at $37^{\circ} \mathrm{C}$ under $5 \% \mathrm{CO}_{2}$ in Dulbecco's modified Eagle's medium (DMEM) supplemented with 10\% fetal bovine serum (FBS) and 1\% penicillin-streptomycin-glutamine. Near-confluent cell culture monolayers were produced by seeding 200000 cells on $35 \mathrm{~mm}$ petri dishes. The dishes were fixed $24 \mathrm{~h}$ later in ice-cold methanol for 5 min.

\subsection{Dorsal root ganglion (DRG) explant samples}

Individual dorsal root ganglia were harvested from E7-E10 chicken embryos undergoing normal development in eggs purchased from Michigan State University Poultry 
Farm. The ganglia were dissected from the dorsal spinal cord in Hank's balanced salt solution (HBSS) containing $1 \%$ anti-anti solution. The ganglia were then seeded on poly-D-lysine-coated $35 \mathrm{~mm}$ polystyrene dishes in DMEM containing $10 \%$ FBS, NGF (100 ng/mL), and $1 \%$ anti-anti solution. The explant cultures were maintained for an additional 7 days with half of the medium replaced every other day. At the end of the culture period, the explants were fixed in $4 \%$ paraformaldehyde for $10 \mathrm{~min}$.

\subsection{Aorta sections}

Sprague-Dawley rats (1 - to 2-month-old males) were euthanized by $\mathrm{CO}_{2}$ inhalation followed by bilateral thoracotomy. The abdominal aortas were immediately dissected and fixed in formalin overnight. All procedures involving animals were approved by the University of Michigan University Committee on Use and Care of Animals. The aortas were embedded in paraffin and sectioned by the University of Michigan Histology Core. The sections were deparaffinized by sequential $2 \mathrm{~min}$ rinses in xylene (twice), 1:1 xylene:ethanol, 100\% ethanol (twice), 95\% ethanol, 70\% ethanol, 50\% ethanol, and distilled water. Antigen recovery was performed by incubating the deparaffinized sections in citrate buffer ( $\mathrm{pH}$ 6.0) containing $0.05 \%$ Triton $\mathrm{X}-100$ at $\sim 100^{\circ} \mathrm{C}$ for $20 \mathrm{~min}$.

\subsection{Aqueous two-phase systems (ATPSs)}

Solutions of 10\% PEG (MW 35000 g/mol; Sigma, St. Louis, MO) containing $0.1 \%$ bovine serum albumin ( $96 \%$ purity; Sigma) and 10\% dextran (MW 500000 g/mol; Pharmacosmos, Holbaek, Denmark) were used to form aqueous twophase systems (ATPSs). The PEG solutions were applied to the samples at volumes sufficient to completely cover the samples (usually $2 \mathrm{~mL}$ for $35 \mathrm{~mm}$ petri dishes or up to $20 \mathrm{~mL}$ for $75 \mathrm{~mm} \times 25 \mathrm{~mm}$ glass slide contained within larger vessels). The antibodies were diluted in the dextran solutions and applied to the samples by micropipetting, either using handheld micropipettes or a previously described pneumatic dispensing system $[10,13]$, in volumes of $0.1-2 \mu \mathrm{L}$.

\subsection{Immunostaining procedures}

For immunofluorescence detection, the samples were blocked immediately after fixation for $1 \mathrm{~h}$ in $10 \%$ normal goat serum. After blocking, the samples were completely covered with PEG and the primary antibodies were applied by micropipetting dextran/antibody droplets $(0.1-2 \mu \mathrm{L}$ in volume) onto the surface of the samples. The samples were then incubated overnight at $4^{\circ} \mathrm{C}$. The next day, the PEG and dextran solutions were thoroughly washed away through three rapidly applied washes and two 5-min washes in PBS. The appropriate secondary antibodies were then bath applied to the samples for $2 \mathrm{~h}$ at room temperature in the dark. Finally, three 5-min washes in PBS were performed and the samples were mounted for imaging. The following primary antibodies were used at various dilutions: rabbit-anti-histone $2 \mathrm{~B}$, mouse-anti-cytokeratin 7, mouse-anti-CDH1 (E-cadherin [ECad]), mouse-anti-Tuj1, mouse-anti- $\alpha$-smooth muscle actin (SMA), and FITC-conjugated rabbit-anti-rat IgG (all from Sigma). Alexa-594-goat-anti-mouse IgG, Alexa-594goat-anti-rabbit IgG, and Alexa-488-goat-anti-mouse IgG secondary antibodies (all from Life Technologies, Carlsbad, CA) were used at 1:500 dilutions. For the dorsal root ganglion (DRG) explants, fluorescein isothiocyanatewheat germ agglutinin (FITC-WGA) $(10 \mu \mathrm{g} / \mathrm{mL}$ in dextran; Life Technologies) was visualized after incubation for $2 \mathrm{~h}$ at room temperature in the dark, followed by washing in PBS as described above. Tetramethylrhodamine isothiocyanate (TRITC)-dextran $(10 \mu \mathrm{g} / \mathrm{mL}$ in dextran; Life Technologies) was sometimes used to visualize the dextran droplets before washing.

For chromogenic and chemiluminescent detection, the samples were first blocked for endogenous peroxidase activity for $1 \mathrm{~h}$ in $1 \% \mathrm{H}_{2} \mathrm{O}_{2}$ and then blocked with $10 \%$ normal goat serum for $1 \mathrm{~h}$. The primary antibodies were applied and the samples were thoroughly washed as described above. Biotinylated (or in some cases horse radish peroxidase [HRP]-conjugated) secondary antibodies were then bath applied for $2 \mathrm{~h}$ at room temperature. The samples were then washed three times in PBS, before streptavidin-HRP (R\&D Systems, Minneapolis, MN) was applied for $45 \mathrm{~min}$. Finally, the samples were washed again and the chromogenic and chemiluminescent signals were developed using diaminobenzidine (Life Technologies) and SuperSignal Femto reagent (Thermo Scientific, Waltham, $\mathrm{MA}$ ), respectively. It is important to note that it would also be possible to detect antigens using biotin-conjugated primary antibodies. Although, we did not test this in the present study, our previous studies using ELISAs demonstrate that direct detection of antigens by way of chemiluminescence is possible using ATPSs. The following antibodies were used: rabbit-anti-histone 2B (Sigma), mouse-anticytokeratin 7 (Sigma) and mouse-anti-CDH1 (ECad) (Sigma), biotin-goat-anti-mouse (Life Technologies), and HRPgoat-anti-rabbit (Santa Cruz Biotechnology, Dallas, TX).

\subsection{Imaging and microscopy}

A Nikon TE300 microscope was used for brightfield and fluorescence imaging. A Fluorchem $\mathrm{M}$ Western reader was used for chemiluminescence detection. Images of samples developed with diaminobenzidine were acquired using a handheld digital camera.

\section{Results}

ATPS-mediated multiplexed immunostaining can be used to pattern primary antibodies on cells in a variety of 

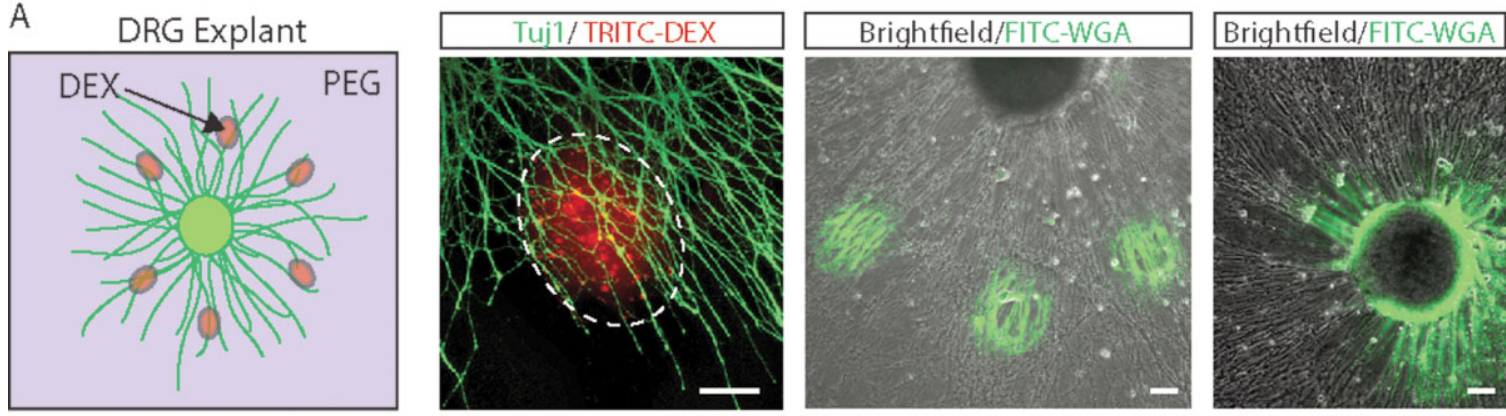

B

Aorta Cross-Section
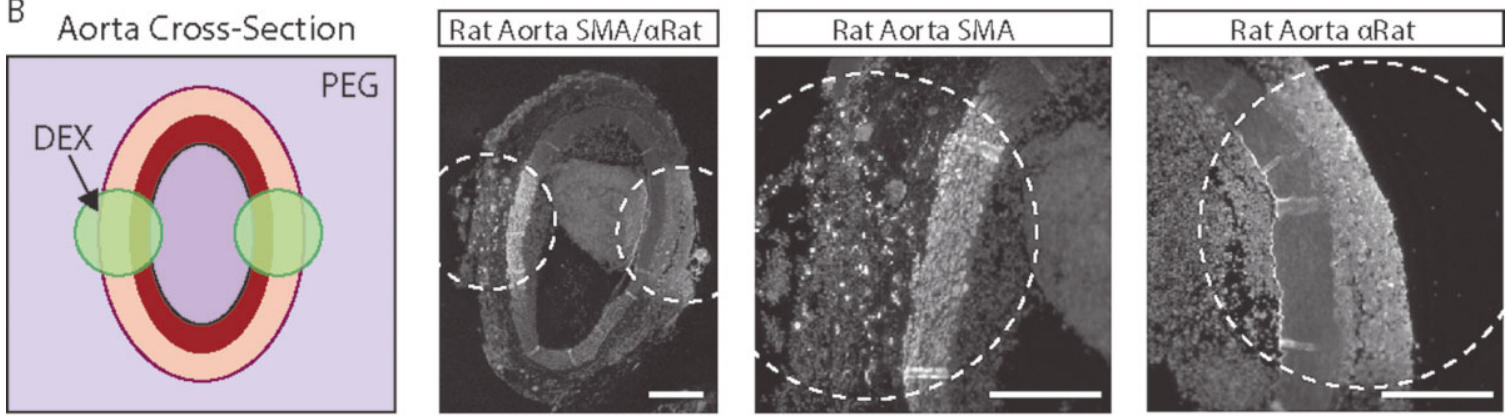

Figure 2. Multiplexed immunostaining of tissue explants and histological sections. (A) DRG explants from chick embryos were selected to demonstrate the potential of this technique for multiplexed immunostaining of complex samples. The micropatterning system was initially tested by localizing TRITC-dextran-containing dextran microdroplets to the axons of Tuj1-labeled DRG explants (left two images). We next tested the ability to micropattern biochemical stains, such as FITC-wheat germ agglutinin (FITC-WGA). FITC-WGA staining could be used to selectively label both the axons and ganglia of the DRG explants (right two images). Scale bars are $100 \mu \mathrm{m}$. (B) Multiplexed immunostaining of histological section was demonstrated on paraffinized cross-sections of rat abdominal aortas using anti- $\alpha$-smooth muscle actin (SMA) and anti-rat antibodies. Scale bars are $\sim 200 \mu \mathrm{m}$.

patterns, as evidenced by the formation of an immunostained "Michigan M" on a HeLa cell monolayer (Fig. 1B, top). Using a gel loading micropipette tip attached to a pneumatic dispensing pump, we were able to generate droplets of dextran in $\mathrm{PEG}$ ranging from $0.8 \mu \mathrm{L}$ for a $0.005 \mathrm{~s}$ air pulse to $4 \mu \mathrm{L}$ for a $0.0175 \mathrm{~s}$ air pulse. We previously demonstrated that a capillary needle can be used to produce much smaller droplets as small as 10 pL. Using handheld micropipettes, it is possible to produce droplets as small as $100 \mathrm{~nL}$. In all of these dispensing systems, the maximum droplet volume is determined by the maximum amount of dextran solution that can be held in the dispensing tip, although generally, smaller droplets ( $1 \mathrm{~nL}$ to $2 \mu \mathrm{L}$ range) are preferred for micropatterning applications. We previously demonstrated that dextran droplets remain stable, without substantial changes to their size or shape during incubation $[9,11]$.

We used HeLa and MCF7 cell monolayers to demonstrate that ATPS-mediated antibody micropatterning strategy enables rapid and cost-efficient optimization of primary antibody concentrations (Fig. 1B, middle and bottom). We tested three concentrations of three antibodies raised against histone $2 \mathrm{~B}$ (H2B, nuclear localization), cytokeratin 7 (CK7, cytoskeletal localization), and ECad (cell membrane localization). It is known that HeLa cells express CK7, but not ECad; whereas MCF7 cells express
ECad, but not CK7. Both cell types express H2B. As expected, we observed a decrease in chemiluminescence with decreasing concentrations of all three antibodies (quantification shown in Supporting information, Fig. S1).

Also as expected, HeLa cells stained intensely for CK7, with faint ECad staining only at the highest concentration of ECad antibody. This low level of signal at the 1:100 dilution of anti-ECad antibody was likely due to non-specific binding, since proper cell-cell junction localization of ECad signal was not observed for HeLa cells. In contrast, the MCF7 cells did not stain for CK7 at any of the antibody concentrations, but clearly stained for ECad. Based on this experiment, one can select the most appropriate antibody dilutions for further experiments. The appropriate subcellular localizations of these three markers are shown in the fluorescence images in Fig. 1C. In addition, we demonstrated that the ATPS-mediated antibody micropatterning strategy is appropriate for use with the most common modes of detection including chromogenic detection (Supporting information, Fig. S2), chemiluminescent detection (Fig. 1B), and immunofluorescence (Fig. 1C). We also noted that the signal decreased at shorter incubation times, as tested using chromogenic detection of CK7 (Supporting information, Fig. S3).

We next demonstrated that ATPS-mediated antibody micropatterning approach can be used to stain more com- 
plex samples such as DRG explants (Fig. 2A) and rat abdominal aorta sections (Fig. 2B). For even more precise patterning, we delivered the dextran droplets using a previously described capillary needle pipetting system [10, 13]. We were able to deliver dextran droplets selectively to the DRG axon terminals, as indicated by the localization of TRITC-dextran to the axons of Tuj1-stained DRG explants. We demonstrated biochemical staining with FITC-WGA by selectively staining the axons (Fig. 2A, third column) and ganglia (Fig. 2A, fourth column) of the explants. Finally, we demonstrated immunohistochemical staining of fixed sections of rat abdominal aorta using two markers: $\alpha$-SMA and FITC-anti-rat. As expected, the SMA antibody primarily labeled the tunica media of the aorta cross-sections, while the anti-rat antibody labeled the tunica intima, media, and adventitia, as well as the thrombus in the lumen.

\section{Discussion}

We demonstrate the strength of the ATPS-mediated antibody micropatterning system for multiplexed labeling of cell cultures, explants, and tissue sections. In comparison to conventional immunostaining protocols that require milliliter volumes of antibody solutions to cover each sample, the ATPS-mediated antibody micropatterning system vastly reduces the amount of reagents required, using diluted antibody volumes of $0.1-2 \mu \mathrm{L}$. In addition, the ATPS-mediated antibody micropatterning technique allows multiple antigens to be probed within the same sample and read out in a single channel. In contrast to other methods for multiplexed immunostaining [3-7], the ATPS-mediated antibody micropatterning method does not require any specialized equipment or non-standard reagents. In fact, it follows a workflow identical to the standard workflow for immunostaining, with the exception of the ATPS micropatterning step.

ATPS-mediated antibody micropatterning provides several benefits to researchers and clinicians. First, there is no optical cross-talk or antibody cross-reactivity that can confound interpretation of the results because the antibodies are retained at separate addressable sites on the sample. Second, the results can be read using a single channel, facilitating more rapid data collection and analysis using a variety of detection modalities. Finally, in select cases where tissue sections or cells are in short supply, ATPS-mediated antibody micropatterning can facilitate more judicious sample usage. Because the ATPS-mediated antibody micropatterning technique adopts standard immunostaining work flows and easily accessed reagents and tools, we expect it to be easily adopted by biomedical researchers and clinics.
The authors would like to acknowledge funding from the Coulter Foundation Grant, NIH (CA 170198) and a generous gift from the Beyster Foundation. J.B.W. thanks the NSF (DGE 0718128; ID: 2010101926) for a pre-doctoral fellowship. J.P.F. designed and performed experiments and wrote the paper, M.T. performed experiments and assisted with writing the methods sections, J.B.W. performed the chromogenic detection experiments and assisted with writing the main text, A.T.A. assisted with experiments and S.T. supervised the project.

Conflicts of interest: J.B.W and S.T. own stock in PHASIQ, Inc. a company working on related technologies.

\section{References}

[1] Brandtzaeg, P., The increasing power of immunohistochemistry and immunocytochemistry. J. Immunol. Methods 1998, 216, 49-67.

[2] Matos, L. L., Trufelli, D. C., de Matos, M. G., da Silva Pinhal, M.A., Immunohistochemistry as an important tool in biomarkers detection and clinical practice. Biomarker Insights 2010, 5, 9-20.

[3] Xing, Y., Chaudry, Q., Shen, C., Kong, K. Y. et al., Bioconjugated quantum dots for multiplexed and quantitative immunohistochemistry. Nat. Protoc. 2007, 2, 1152-1165.

[4] Gerdes, M. J., Sevinsky, C. J., Sood, A., Adak, S. et al., Highly multiplexed single-cell analysis of formalin-fixed, paraffin-embedded cancer tissue. Proc. Natl. Acad. Sci. USA 2013, 110, 11982-11987.

[5] Gannot, G., Tangrea, M. A., Erickson, H. S., Pinto, P. A. et al., Layered peptide array for multiplex immunohistochemistry. J. Mol. Diagn. 2007, 9, 297-304.

[6] Lovchik, R. D., Kaigala, G. V., Georgiadis, M., Delamarche, E., Microimmunohistochemistry using a microfluidic probe. Lab Chip 2012, 12, 1040-1043.

[7] Kim, M. S., Kim, T., Kong, S. Y., Kwon, T. et al., Breast cancer diagnosis using a microfluidic multiplexed immunohistochemistry platform. PLoS ONE 2010, 5, e10441.

[8] Albertsson, P. A., Partition of Cell Particles and Macromolecules: Separation and Purification of Biomolecules, Cell Organelles, Membranes, and Cells in Aqueous Polymer Two-Phase Systems and Their Use in Biochemical Analysis and Biotechnology, 3rd Edn., Wiley, New York 1986, pp. 346.

[9] Tavana, H., Jovic, A., Mosadegh, B., Lee, Q. Y. et al., Nanolitre liquid patterning in aqueous environments for spatially defined reagent delivery to mammalian cells. Nat. Mater. 2009, 8, 736-741.

[10] Frampton, J. P., Shi, H., Kao, A., Parent, J. M. et al., Delivery of proteases in aqueous two-phase systems enables direct purification of stem cell colonies from feeder cell co-cultures for differentiation into functional cardiomyocytes. Adv. Healthc. Mater. 2013, 2, 1440-1444.

[11] Frampton, J. P., White J. B., Simon, A. B., Tsuei M. et al., Aqueous two-phase system patterning of detection antibody solutions for cross-reaction-free multiplexed ELISA. Sci. Rep. 2014, 4, 4878.

[12] Simon, A. B., Frampton, J. P., Huang, N. T., Kurabayashi, K. et al., Aqueous two-phase systems enable multiplexing of homogeneous immunoassays. Technology 2014, DOI:10.1142/S2339547814500150.

[13] Frampton, J. P., Fang, Z. Z., Simon, A. B., Chen D. et al., Aqueous two-phase system patterning of microbubbles: Localized induction of apoptosis in sonoporated cells. Adv. Funct. Mater. 2013, 23, 3420-3431. 


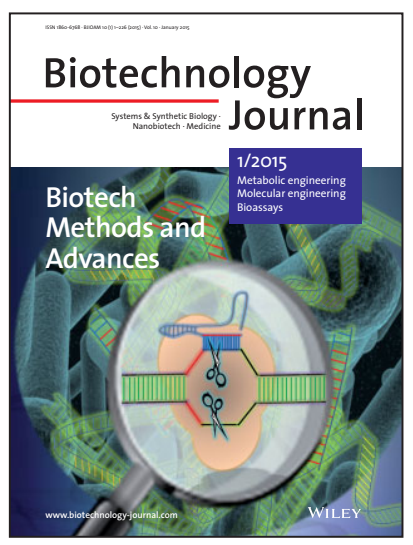

Special issue: Methods and Advances. Each year Biotechnology Journal kicks off with the special Methods and Advances issue. This issue includes novel methods for fluorescent detection of target molecules, cellular transfection and arrays as well as state-of-the-art reviews on lipases, cell-free metabolic engineering, stem cells and tissue engineering and more. Chan Woo Song, Joungmin Lee and Sang Yup Lee review recent advances for genome engineering and gene expression control in various bacteria. The cover is a graphical representation of one of genome editing tools employing the CRISPR-Cas system. Image by Sang Yup Lee. See the article: http://dx.doi.org/10.1002/biot.201400057

\section{Biotechnology Journal - list of articles published in the January 2015 issue.}

Editorial: Methods and Advances -

Biotech progress for science and our daily lives

Sang Yup Lee and Alois Jungbauer

http://dx.doi.org/10.1002/biot.201400842

Editorial: Looking back and looking forward 2014 and 2015 in Biotechnology journal

Jing Zhu and Uta Göbel

http://dx.doi.org/10.1002/biot.201400820

Review

Effects of methanol on lipases: Molecular, kinetic and process issues in the production of biodiesel

Marina Lotti, Jürgen Pleiss, Francisco Valero and Pau Ferrer

http://dx.doi.org/10.1002/biot.201400158

Review

Novel lipase purification methods -

a review of the latest developments

Chung Hong Tan, Pau Loke Show, Chien Wei Ooi,

Eng-Poh Ng, John Chi-Wei Lan and Tau Chuan Ling

http://dx.doi.org/10.1002/biot.201400301

Review

Universal probe amplification: Multiplex screening technologies for genetic variations

Jung Hun Park, Ki Soo Park, Kyungmee Lee, Hyowon Jang and Hyun Gyu Park

http://dx.doi.org/10.1002/biot.201400219

Review

Genome engineering and gene expression control

for bacterial strain development

Chan Woo Song, Joungmin Lee and Sang Yup Lee

http://dx.doi.org/10.1002/biot.201400057

Review

Cell-free metabolic engineering: Biomanufacturing beyond the cell

Quentin M. Dudley, Ashty S. Karim and Michael C. Jewett

http://dx.doi.org/10.1002/biot.201400330

\section{Review}

Human pluripotent stem cell-derived products: Advances towards robust, scalable and cost-effective manufacturing strategies

Michael J. Jenkins and Suzanne S. Farid

http://dx.doi.org/10.1002/biot.201400348

\section{Review}

Nanoarchitecture of scaffolds and endothelial cells in engineering small diameter vascular grafts Krishna Kumar Sankaran, Anuradha Subramanian, Uma Maheswari Krishnan and Swaminathan Sethuraman http://dx.doi.org/10.1002/biot.201400415

\section{Review}

Improving livestock for agriculture - technological progress from random transgenesis to precision genome editing heralds a new era

Götz Laible, Jingwei Wei and Stefan Wagner

http://dx.doi.org/10.1002/biot.201400193

Biotech Methods

Aqueous two-phase system-mediated antibody micropatterning enables multiplexed immunostaining of cell monolayers and tissues

John P. Frampton, Michael Tsuei, Joshua B. White,

Abin T. Abraham and Shuichi Takayama

http://dx.doi.org/10.1002/biot.201400271

Biotech Method

Catalytically impaired fluorescent Class $C \beta$-lactamase enables rapid and sensitive cephalosporin detection by stabilizing fluorescence signals: Implications for biosensor design Man-Wah Tsang, Pui-Kin So, Sze-Yan Liu, Chun-Wai Tsang, Pak-Ho Chan, Kwok-Yin Wong and Yun-Chung Leung http://dx.doi.org/10.1002/biot.201400140

Biotech Method

Adenosine reagent-free detection by co-immobilization of adenosine deaminase and phenol red on an optical biostrip Foteini Bartzoka, Katerina Venetsanou and Yannis Clonis http://dx.doi.org/10.1002/biot.201400333 
Biotech Method

A combination of targeted toxin technology and the piggyBacmediated gene transfer system enables efficient isolation of stable transfectants in nonhuman mammalian cells Masahiro Sato, Emi Inada, Issei Saitoh, Yuko Matsumoto, Masato Ohtsuka, Hiromi Miura, Shingo Nakamura, Takayuki Sakurai and Satoshi Watanabe

http://dx.doi.org/10.1002/biot.201400283

Biotech Method

The LQSP tetrapeptide is a new highly efficient substrate of microbial transglutaminase for the site-specific derivatization of peptides and proteins

Andrea Caporale, Fabio Selis, Annamaria Sandomenico, Gloria S. Jotti, Giancarlo Tonon and Menotti Ruvo

http://dx.doi.org/10.1002/biot.201400466

Biotech Method

Representative mammalian cell culture test materials for assessment of primary recovery technologies: A rapid method with industrial applicability

Daria Popova, Adam Stonier, David Pain, Nigel J. TitchenerHooker and Suzanne S. Farid

http://dx.doi.org/10.1002/biot.201400294

Research Article

Fluorescence techniques used to measure interactions between hydroxyapatite nanoparticles and epidermal growth factor receptors

Mustafa H. Kathawala, Stella P. K. Khoo,

Thankiah Sudhaharan, Xinxin Zhao, Joachim Say Chye Loo,

Sohail Ahmed and Kee Woei Ng

http://dx.doi.org/10.1002/biot.201400404

Research Article

(+)-Valencene production in Nicotiana benthamiana

is increased by down-regulation of competing pathways

Katarina Cankar, Esmer Jongedijk, Martin Klompmaker,

Timotej Majdic, Roland Mumm, Harro Bouwmeester,

Dirk Bosch and Jules Beekwilder

http://dx.doi.org/10.1002/biot.201400288
Research Article

High-throughput nucleoside phosphate monitoring in mammalian cell fed-batch cultivation using quantitative matrix-assisted laser desorption/ionization time-of-flight mass spectrometry

Robert F. Steinhoff, Marija Ivarsson, Tobias Habicher, Thomas K. Villiger, Jens Boertz, Jasmin Krismer,

Stephan R. Fagerer, Miroslav Soos, Massimo Morbidelli, Martin Pabst and Renato Zenobi

http://dx.doi.org/10.1002/biot.201400292

Research Article

Glycoarrays with engineered phages displaying structurally diverse oligosaccharides enable high-throughput detection of glycan-protein interactions

Eda Çelik, Anne A. Ollis, Yi Lasanajak, Adam C. Fisher, Göksu Gür, David F. Smith and Matthew P. DeLisa

http://dx.doi.org/10.1002/biot.201400354

Research Article

Xylan degradation improved by a combination of monolithic columns bearing immobilized recombinant $\beta$-xylosidase from Aspergillus awamori X-100 and Grindamyl $\mathrm{H} 121 \beta$-xylanase Maria V. Volokitina, Kirill S. Bobrov, Kathleen Piens, Elena V. Eneyskaya, Tatiana B. Tennikova, Evgenia G. Vlakh and Anna A. Kulminskaya

http://dx.doi.org/10.1002/biot.201400417

Rapid Communication

Site-specific conjugation of an antibody-binding protein catalyzed by horseradish peroxidase creates a multivalent protein conjugate with high affinity to IgG

Kosuke Minamihata, Masahiro Goto, and Noriho Kamiya

http://dx.doi.org/10.1002/biot.201400512 